\title{
Editorial: Recent Empirical Research and Methodologies in Defense Mechanisms: Defenses as Fundamental Contributors to Adaptation
}

\author{
Mariagrazia Di Giuseppe ${ }^{1 *}$, John Christopher Perry ${ }^{2}$, Tracy A. Prout ${ }^{3}$ and \\ Ciro Conversano ${ }^{1}$
}

${ }^{1}$ Department of Surgical, Medical and Molecular Pathology, Critical and Care Medicine, University of Pisa, Pisa, Italy, ${ }^{2}$ McGill University and the Institute of Community and Family Psychiatry, Jewish General Hospital, Montreal, QC, Canada, ${ }^{3}$ Ferkauf Graduate School of Psychology, Yeshiva University, Bronx, NY, United States

Keywords: defense mechanisms, adaptation, mental disorder, distress, assessment, DMRS

\section{Editorial on the Research Topic}

\section{Recent Empirical Research and Methodologies in Defense Mechanisms}

During the past 50 years, empirical research on defense mechanisms has come a long way in contributing to the empirical science. Beginning with Freud's initial description of defenses (Freud, 1894), there have been numerous suggested revisions to the theory of defenses. At this juncture,

\section{OPEN ACCESS}

Edited and reviewed by: Antoine Bechara, University of Southern California, United States

*Correspondence: Mariagrazia Di Giuseppe mariagrazia.digiuseppe@gmail.com

Specialty section: This article was submitted to Psychopathology, a section of the journa

Frontiers in Psychology

Received: 26 October 2021 Accepted: 28 October 2021 Published: 03 December 2021

Citation: Di Giuseppe M, Perry JC, Prout TA and Conversano C (2021) Editorial:

Recent Empirical Research and Methodologies in Defense Mechanisms: Defenses as

Fundamental Contributors to Adaptation.

Front. Psychol. 12:802602 doi: 10.3389/fpsyg.2021.802602 there is general agreement on the hierarchical organization of defense mechanisms, which, for instance lead to the inclusion of an Provisional Axis for Defensive Functioning in the 4th Edition of the Diagnostic and Statistical Manual for Mental Disorders (American Psychiatric Association, 1994). The impact of defensive functioning on physical and psychological well-being has been widely demonstrated. Less appreciated than research on defenses and mental disorders, are the growing body of studies of defenses and medical conditions (Martino et al., 2019, 2020; Conversano and Di Giuseppe, 2021). Recent studies have found that cancer patients with self-sacrificing defensive style had shorter disease-free intervals, shorter survival times, and a more unfavorable cancer staging at endpoint (Weihs et al., 2000; Di Giuseppe et al., 2018). Other studies (Zilikis and Dervenis, 2003; Vita et al., 2020; Marchini et al., 2021) have linked repressed conflicts to physical and mental disorders among those with a recurrent history of unexplained distress and depression. In line with the psychosomatic hypothesis, using neurotic defenses, which inhibit awareness of disturbing wishes, feelings, thoughts, experiences and memories, lead to impaired endocrine and immune functions and relate to the somatic manifestation of psychological distress (Bahnson and Bahnson, 1966; Vos and de Haes, 2007). Moreover, defense mechanisms, like other emotion regulation strategies, function as essential moderators of psychological responses to stressful life events (Conversano et al., 2020; Di Giuseppe et al., 2021; Merlo et al.) such as chronic physical and mental conditions (Perry, 1988; Sardella et al., 2021; Martino et al.; Martino et al.).

Defenses operate largely or partly outside of awareness, and their effects take innumerable forms. Both of these realities have created measurement challenges. Some measures (Gleser and Ihilevich, 1969; Plutchik et al., 1979; Bond et al., 1983) may have been based on the same motivational constructs but presented different organizing schemas (Horowitz et al., 1992). Other measures (Cramer, 1991; Lerner, 2005) have yielded scientifically valuable findings (Cramer, 2015), but limited themselves to a small number of defenses and lack of clear link to how individuals cope with internal and external conflicts and stressors (Rosso et al.). These methodological limitations have contributed for years to the confusion between defense mechanisms and coping strategies. 
As Cramer stated "Coping mechanisms involve a conscious, purposeful effort, while defense mechanisms are processes that occur without conscious effort and without conscious awareness (i.e., they are unconscious). Also, coping strategies are carried out with the intent of managing or solving a problem situation, while defense mechanisms occur without conscious intentionality; the latter function to change an internal psychological state but may have no effect on external reality, and so may result in non-veridical perception, that is, in reality distortion" (Cramer, 1998, p. 921). With continued advances in empirical research on the hierarchical nature of defense adaptation, two aspects of Cramer's definition appear incomplete. First, some defenses confuse internal conflict with external stress and lead the individual to maladaptive responses to the environment (e.g., counter-attacking rather than reflecting before acting). Second, it does not capture the partially conscious and more flexibly adaptive aspects of mature defensive functioning.

Defense mechanisms higher in the hierarchy (i.e., high adaptive defenses) do not follow the differential criteria described in Cramer's theory (Beresford, 2012). More than 30 years research with the Defense Mechanisms Rating Scales (DMRS; Perry, 1990) and its derivative measures (DMRS-Q; Di Giuseppe et al., 2014; Di Giuseppe and Perry, 2021; DMRS-SR-30; Di Giuseppe et al., 2020a) have demonstrated that individuals using mature defenses can: (1) be partially or fully aware of their activation (e.g., altruism, self-assertion, or self-observation); (2) intentionally use an adaptive defensive strategy to deal with internal conflict or stressful situations (e.g., anticipation or suppression); and (3) increase the probability of a gratifying resolution of the internal or external stressors without reality distortion (e.g., selfassertion or sublimation). In light of these findings, we affirm that the hierarchy of defense mechanisms (Vaillant, 1992; American Psychiatric Association, 1994; Perry, 2014; Di Giuseppe and Perry) is a comprehensive description of both more adaptive (i.e., mature defenses, overlapping in function with coping strategies) and less adaptive (i.e., immature and neurotic defenses) way of automatically responding. A clear result is that the systematic assessment of defenses which reflects this hierarchy of adaptation adds value to the diagnosis of mental disorders. We believe that it is an essential part (Perry et al., 2020; Conversano, 2021).

Research on personality disorders has found that specific defensive profiles are associated with personality traits and disorders (Maffei et al., 1995; Steiner et al., 2007; Presniak et al., 2010; Perry et al., 2013) and revealed a hierarchical organization of personality disorders based on the maturity of defensive functioning (Di Giuseppe et al., 2019). This confirms Kernberg's Personality Organization, which includes object representations (split vs. ambivalent objects) and reality testing (Kernberg, 1984). As highlighted by Kempe et al. by analyzing the defensive functioning of individuals with narcissistic personality it is possible to distinguish the defensive profiles of both grandiose and vulnerable narcissism (Kempe et al.). Similarly, recent studies investigated defenses in relation to attachment and mentalization show promising results. As demonstrated by Tanzilli et al. (2021), depressed patients with secure attachment showed higher reflective functioning and overall defensive maturity then those with insecure attachment. In line with these findings, Békés et al. found that the use of neurotic and immature defenses in the early phase of treatment predicted an increase in avoidant attachment over the course of treatment, whereas the use of immature non-depressive defenses (e.g., denial, rationalization) predicted a decrease in preoccupied attachment. Similarly, Hayden et al. reported that mentalization played an important role in the reduction of maladaptive defenses during inpatient therapy. However, their results showed that only maladaptive defenses decreased significantly in psychotherapy, while neurotic and mature did not increase significantly as expected. It is possible that some of these contradictory results stem from methodological issues, such as employing measures with inadequate reliability and validity. Furthermore, other research has demonstrated that specific defense are associated with psychiatric symptoms, interpersonal problems, externalizing behaviors, vulnerable sense of self, poor adjustment, suicidal ideation, and attempts (Dell'Osso et al., 2011; Boldrini et al., 2020).

Psychotherapy is important to help individuals improve their defensive functioning and outcome (Hoffman et al., 2016; Babl et al., 2019; Di Giuseppe et al., 2020b; Hersoug et al.). The proportion of mature and immature defenses change during psychotherapy and predict treatment response (Perry and Bond, 2012; Perry et al., 2020; Prout et al., 2021; Beresford et al.; de Roten et al.). Patient improvement in defensive maturity is likely to happen within the relationship with the therapist, who in turn activates and works with defenses in response to stress (Tanzilli and Gualco, 2020; Tanzilli et al., 2020). Therapists appear to utilize higher levels of mature defenses and lower levels of immature defenses compared to a community sample (Aafjes-van Doorn et al.). However, lower therapists' defensive maturity was associated with higher levels of vicarious trauma and professional doubt during the COVID-19 pandemic (Aafjes-van Doorn et al.). Further studies should investigate the impact of the interplay between patient and therapist defense mechanisms on outcome.

In addition to psychopathology research, interesting findings come from a number of recent studies conducted on general populations under stressful conditions. Maladaptive defensive responses were reported more often by younger people during the first wave of COVID-19 pandemic, while greater reliance on mature defenses was evident among older adults during the pandemic (Prout et al., 2020). These findings were confirmed by Beresford et al. who found that maturity of defensive functioning was associated with older age and it predicted lower depression levels in a large sample of adult cancer patients. Defense mechanisms are also associated with gender. Women use more neurotic and immature-depressive defenses to deal with internal or external stressors, while in similar conditions men tend to rely more on obsessional and immature-non depressive defenses (see Di Giuseppe and Perry, 2021, for review on the hierarchy of defenses). These findings were confirmed also in individuals diagnosed with gender dysphoria, indicating that the individual's dominant defensive functioning is related to the gender to which one chooses instead of the gender assigned at birth (Giovanardi et al.). 
Advances in research on defense mechanisms widely demonstrate the impact of defense mechanisms in the onset, course, and amelioration of mental disorders. Despite the increasingly robust findings that defense mechanisms, and the hierarchy of adaptation, add scientific value to diagnosis, many practitioners have limited awareness of these scientific contributions. This special issue of Frontiers is dedicated to increase awareness of the relevance of these constructs to clinical practice. Although defense mechanisms are included in the Psychodynamic Diagnostic Manual as a key construct in diagnosis and personality structure (Lingiardi and Bornstein, 2017), we believe that greater understanding of patients' defensive functioning will be helpful to non-dynamically oriented clinicians as well. The science of our profession can be improved and the quality of our interventions can be individually tailored as a result of increased research on

\section{REFERENCES}

American Psychiatric Association (1994). Diagnostic and Statistical Manual of Mental Disorders, 4th Edn. Washington, DC: American Psychiatric Publishing Inc.

Babl, A., grosse Holtforth, M., Perry, J. C., Schneider, N., Dommann, E., Heer, S., et al. (2019). Comparison and change of defense mechanisms over the course of psychotherapy in patients with depression or anxiety disorder: evidence from a randomized controlled trial. J. Affect. Disord. 252, 212-220. doi: 10.1016/j.jad.2019.04.021

Bahnson, C. B., and Bahnson, M. B. (1966). Role of the ego defenses: denial and repression in the etiology of malignant neoplasm. Ann. N. Y. Acad. Sci. 125, 827-845. doi: 10.1111/j.1749-6632.1966.tb45434.x

Beresford, M. D. (2012). Psychological Adaptive Mechanisms: Ego Defense Recognition in Practice and Research. New York, NY: Oxford University Press.

Boldrini, T., Lo Buglio, G., Giovanardi, G., Lingiardi, V., and Salcuni, S. (2020). Defense mechanisms in adolescents at high risk of developing psychosis: an empirical investigation. Res. Psychothera. 23, 4-15. doi: 10.4081/ripppo.2020.456

Bond, M., Gardner, S. T., Christian, J., and Sigal, J. J. (1983). Empirical study of self-rated defense styles. Arch. Gen. Psychiatry 40, 333-338. doi: 10.1001/archpsyc.1983.01790030103013

Conversano, C. (2021). The psychodynamic approach during COVID-19 emotional crisis. Front. Psychol. 12:670196. doi: 10.3389/fpsyg.2021.670196

Conversano, C., Ciacchini, R., Orr,ù, G., Di Giuseppe, M., Gemignani, A., and Poli, A. (2020). Mindfulness, compassion, and self-compassion among health care professionals: what's new? A systematic review. Front. Psychol. 11:1683. doi: 10.3389/fpsyg.2020.01683

Conversano, C., and Di Giuseppe, M. (2021). Psychological factors as determinants of chronic conditions: clinical and psychodynamic advances. Front. Psychol. 12:635708. doi: 10.3389/fpsyg.2021.635708

Cramer, P. (1991). "The defense mechanism manual," in The Development of Defense Mechanisms. New York, NY: Springer. doi: 10.1007/978-1-4613-9025-1

Cramer, P. (1998). Coping and defense mechanisms: what's the difference?. J. Pers. Assess. 66, 919-946. doi: 10.1111/1467-6494.00037

Cramer, P. (2015). Defense mechanisms: 40 years of empirical research. J. Pers. Assess. 97, 114-122. doi: 10.1080/00223891.2014.947997

Dell'Osso, L., Carmassi, C., Rucci, P., Ciapparelli, A., Conversano, C., and Marazziti, D. (2011). Complicated grief and suicidality: the impact of subthreshold mood symptoms. CNS Spectr. 16, 1-6. doi: 10.1017/ S1092852912000090

Di Giuseppe, M., Ciacchini, R., Micheloni, T., Bertolucci, I., Marchi, L., and Conversano, C. (2018). Defense mechanisms in cancer patients: a systematic review. J. Psychosom. Res. 115, 76-86. doi: 10.1016/j.jpsychores.2018.10.016

Di Giuseppe, M., Gennaro, A., Lingiardi, V., and Perry, J. C. (2019). The role of defense mechanisms in emerging personality disorders in clinical adolescents. Psychiatry 82, 128-142. doi: 10.1080/00332747.2019.1579595 the role of defenses in the course of treatment. Research on defenses adds information about symptom severity, differential diagnosis, treatment compliance, recommended interventions, and expected prognosis. The inclusion of defensive functioning in diagnosis and case formulation (Perry et al., 2018, 2020) has the potential to enrich the overall clinical understanding of patients mental functioning and how treatment can be tailored to meet their needs. In sum, close assessment of defenses provide some of the information needed to develop truly effective, personalized treatments (Zilcha-Mano, 2021).

\section{AUTHOR CONTRIBUTIONS}

All authors contributed in equal part to the Research Topic and the final draft of the manuscript.

Di Giuseppe, M., Nepa, G., Prout, T. A., Albertini, F., Marcelli, S., et al. (2021) Stress, burnout, and resilience among healthcare workers during the COVID19 emergency: the role of defense mechanisms. Int. J. Environ. Res. Public Health 18:5258. doi: 10.3390/ijerph18105258

Di Giuseppe, M., and Perry, J. C. (2021). The hierarchy of defense mechanisms: assessing defensive functioning with the defense mechanisms rating scales Q-sort. Front. Psychol. 12:718440. doi: 10.3389/fpsyg.2021.718440

Di Giuseppe, M., Perry, J. C., Lucchesi, M., Michelini, M., Vitiello, S., Piantanida, A., et al. (2020a). Preliminary reliability and validity of theDMRS-SR-30, a novel self-report based on the defense mechanisms rating scales. Front. Psychiatry 11:870. doi: 10.3389/fpsyt.2020.00870

Di Giuseppe, M., Perry, J. C., Petraglia, J., Janzen, J., and Lingiardi, V. (2014). Development of a Q-sort version of the defense mechanism rating scales (DMRS-Q) for clinical use. J. Clin. Psychol. 70, 452-465. doi: 10.1002/jclp.22089

Di Giuseppe, M., Prout, T. A., Fabiani, M., and Kui, T. (2020b). Defensive profile of parents of children with externalizing problems receiving Regulation Focused Psychotherapy for Children: a pilot study. Mediterranean J. Clin. Psychol. 8. doi: 10.6092/2282-1619/mjcp-2515

Freud, S. (1894). "The neuro-psychoses of defence," in The Standard Edition of the Complete Psychological Works of Sigmund Freud, ed J. Strachey (London: Hogarth Press), 41-61.

Gleser, G. C., and Ihilevich, D. (1969). An objective instrument for measuring defense mechanisms. J. Consult. Clin. Psychol. 33, 51-60. doi: 10.1037/h00 27381

Hoffman, L., Rice, T. R., and Prout, T. A. (2016). Manual of RegulationFocused Psychotherapy for Children (RFP-C) With Externalizing Behaviors: A Psychodynamic Approach. New York, NY: Routledge. doi: 10.4324/ 9781315736648

Horowitz, M., Cooper, S., Fridhandler, B., Perry, J. C., Bond, M., and Vaillant, G. (1992). Control processes and defense mechanisms. J. Psychother. Pract. Res. $1,324-336$.

Kernberg, O. F. (1984). Severe Personality Disorders: Psychotherapeutic Strategies. New Haven, CT; London: Yale University Press.

Lerner, P. M. (2005). "Defense and its assessment: the lerner defense scale," in Scoring the Rorschach: Seven Validated Systems, eds R. F. Bornstein and J. M. Masling (Mahwah, NJ: Lawrence Erlbaum Associates Publishers), 237-269.

Lingiardi, V., and Bornstein, R. F. (2017). "Profile of mental functioning - M axis," in Psychodynamic Diagnostic Manual, 2nd ed., eds V. Lingiardi and N. McWilliams (New York, NY: Guilford Press), 75-133.

Maffei, C., Fossati, A., Lingiardi, V., Madeddu, F., Borellini, C., and Petrachi, M. (1995). Personality maladjustment, defenses, and psychopathological symptoms in nonclinical subjects. J. Pers. Disord. 9, 330-345. doi: 10.1521/pedi.1995.9.4.330

Marchini, F., Langher, V., Napoli, A., Balonan, J. T., Fedele, F., Martino, G., et al. (2021). Unconscious loss processing in diabetes: associations with medication adherence and quality of care. Psychoanal. Psychother. 35, 5-23. doi: 10.1080/02668734.2021.1922492 
Martino, G., Bellone, F., Langher, V., Caputo, A., Catalano, A., Quattropani, and Morabito, N. (2019). Alexithymia and psychological distress affect perceived quality of life in patients with Type 2 diabetes mellitus. Mediterranean J. Clin. Psychol. 7. doi: 10.6092/2282-1619/2019.7.2328

Martino, G., Caputo, A., Vicario, C. M., Catalano, A., Schwarz, P., and Quattropani, M. C. (2020). The relationship between alexithymia and type 2 diabetes: a systematic review. Front. Psychol. 11:2026. doi: 10.3389/ fpsyg.2020.02026

Perry, J. C. (1988). A prospective study of life stress, defenses, psychotic symptoms and depression in borderline and antisocial personality disorders and bipolar type II affective disorder. J. Pers. Disord. 2, 49-59. doi: 10.1521/pedi.198 8.2.1.49

Perry, J. C. (1990). The Defense Mechanism Rating Scales Manual, 5th Edn. Cambridge, MA.

Perry, J. C. (2014). Anomalies and specific functions in the clinical identification of defense mechansims. J. Clin. Psychol. 70, 406-418. doi: 10.1002/jclp.22085

Perry, J. C., Banon, E., and Bond, M. (2020). Change in defense mechanisms and depression in a pilot study of antidepressive medications plus 20 sessions of psychotherapy for recurrent major depression. J. Nerv. Ment. Dis. 208, 261-268. doi: 10.1097/NMD.0000000000001112

Perry, J. C., and Bond, M. (2012). Change in defense mechanisms during long-term dynamic psychotherapy and five-year outcome. Am. J. Psychiatry 169, 916-925. doi: 10.1176/appi.ajp.2012.11091403

Perry, J. C., Knoll, M., and Tran, V. (2018). "Motives, defenses, and conflicts in the dynamic formulation for psychodynamic psychotherapy using the Idiographic Conflict Formulation method," in Case Formulation for Personality Disorders, ed U. Kramer, Chap 11 (Elsevier), 203-224. doi: 10.1016/B978-0-12-813521-1.00011-4

Perry, J. C., Presniak, M. D., and Olson, T. (2013). Defense mechanisms in borderline schizotypal, antisocial and narcissistic personality disorders. Psychiatry 76, 32-52. doi: 10.1521/psyc.2013.76.1.32

Plutchik, R., Kellerman, H., and Conte, H. R. (1979). "A structural theory of ego defenses and emotions," in Emotions in Personality and Psychopathology. Emotions, Personality, and Psychotherapy, ed C. E Izard (Boston, MA: Springer), 227-257. doi: 10.1007/978-1-4613-2892-6_9

Presniak, M. D., Olson, T., and MacGregor, M. W. (2010). The role of defense mechanisms in Borderline and Antisocial personalities. J. Pers. Assess. 92, 137-145. doi: 10.1080/00223890903510373

Prout, T. A., Rice, T., Chung, H., Gorokhovsky, Y., Murphy, S., and Hoffman, L. (2021). Randomized controlled trial of Regulation Focused Psychotherapy for children: a manualized psychodynamic treatment for externalizing behaviors. Psychother. Res. 1-16. doi: 10.1080/10503307.2021.1980626. [Epub ahead of print].

Prout, T. A., Zilcha-Mano, S., Aafjes-van Doorn, K., Békés, V., Christman-Cohen, I., Whistler, K., et al. (2020). Identifying predictors of psychological distress during COVID-19: a machine learning approach. Front. Psychol. 11:586202. doi: 10.3389/fpsyg.2020.586202

Sardella, A., Lenzo, V., Bonanno, G. A., Martino, G., Basile, G., and Quattropani, M. C. (2021). Dispositional optimism and context sensitivity: psychological contributors to frailty status among elderly outpatients. Front. Psychol. 11:621013. doi: 10.3389/fpsyg.2020.621013
Steiner, H., Erickson, S. J., MacLean, P., Medic, S., and Koopman, C. (2007). Relationship between defenses, personality, and affect during a stress task in normal adolescents. Child Psychiatry Hum. Dev. 38, 107-119. doi: 10.1007/s10578-007-0046-9

Tanzilli, A., Di Giuseppe, M., Giovanardi, G., Boldrini, T., Caviglia, G., Conversano, C., et al. (2021). Mentalization, attachment, and defense mechanisms: a psychodynamic diagnostic manual-2-oriented empirical investigation. Res. Psychother. 24:531. doi: 10.4081/ripppo.2021.531

Tanzilli, A., and Gualco, I. (2020). Clinician emotional responses and therapeutic alliance when treating adolescent patients with narcissistic personality disorder subtypes: a clinically meaningful empirical investigation. J. Pers. Disord. 34, 42-62. doi: 10.1521/pedi.2020.34.supp.42

Tanzilli, A., Gualco, I., Baiocco, R., and Lingiardi, V. (2020). Clinician reactions when working with adolescent patients: the therapist response questionnaire for adolescents. J. Pers. Assess. 102, 616-627. doi: 10.1080/00223891.2019.1674318

Vaillant, G. E. (1992). Ego Mechanisms of Defense: A Guide for Clinicians and Researchers. Washington, DC: American Psychiatric Press.

Vita, R., Caputo, A., Quattropani, M. C., Watt, T., Feldt-Rasmussen, U., Puleio, P., et al. (2020). Quality of life in patients with hyperthyroidism: where do we stand? Mediterranean J. Clin. Psychol. 8. doi: 10.6092/2282-1619/mjcp-2521

Vos, M. S., and de Haes, J. C. J. (2007). Denial in cancer patients, an explorative review. Psychooncology. 16, 12-25. doi: 10.1002/pon.1051

Weihs, K. L., Enright, T. M., Simmens, S. J., and Reiss, D. (2000). Negative affectivity, restriction of emotions, and site of metastases predict mortality in recurrent breast cancer. J. Psychosom. Res. 49, 59-68. doi: 10.1016/S0022-3999(00)00143-4

Zilcha-Mano, S. (2021). Toward personalized psychotherapy: the importance of the trait-like/state-like distinction for understanding therapeutic change. Am. Psychol. 76, 516-528. doi: 10.1037/amp0000629

Zilikis, N., and Dervenis, C. (2003). Psychosomatic aspects of pancreatic cancer, Pancreatology 3, 273-275. doi: 10.1159/000071764

Conflict of Interest: The authors declare that the research was conducted in the absence of any commercial or financial relationships that could be construed as a potential conflict of interest.

Publisher's Note: All claims expressed in this article are solely those of the authors and do not necessarily represent those of their affiliated organizations, or those of the publisher, the editors and the reviewers. Any product that may be evaluated in this article, or claim that may be made by its manufacturer, is not guaranteed or endorsed by the publisher.

Copyright (C) 2021 Di Giuseppe, Perry, Prout and Conversano. This is an open-access article distributed under the terms of the Creative Commons Attribution License (CC $B Y)$. The use, distribution or reproduction in other forums is permitted, provided the original author(s) and the copyright owner(s) are credited and that the original publication in this journal is cited, in accordance with accepted academic practice. No use, distribution or reproduction is permitted which does not comply with these terms. 